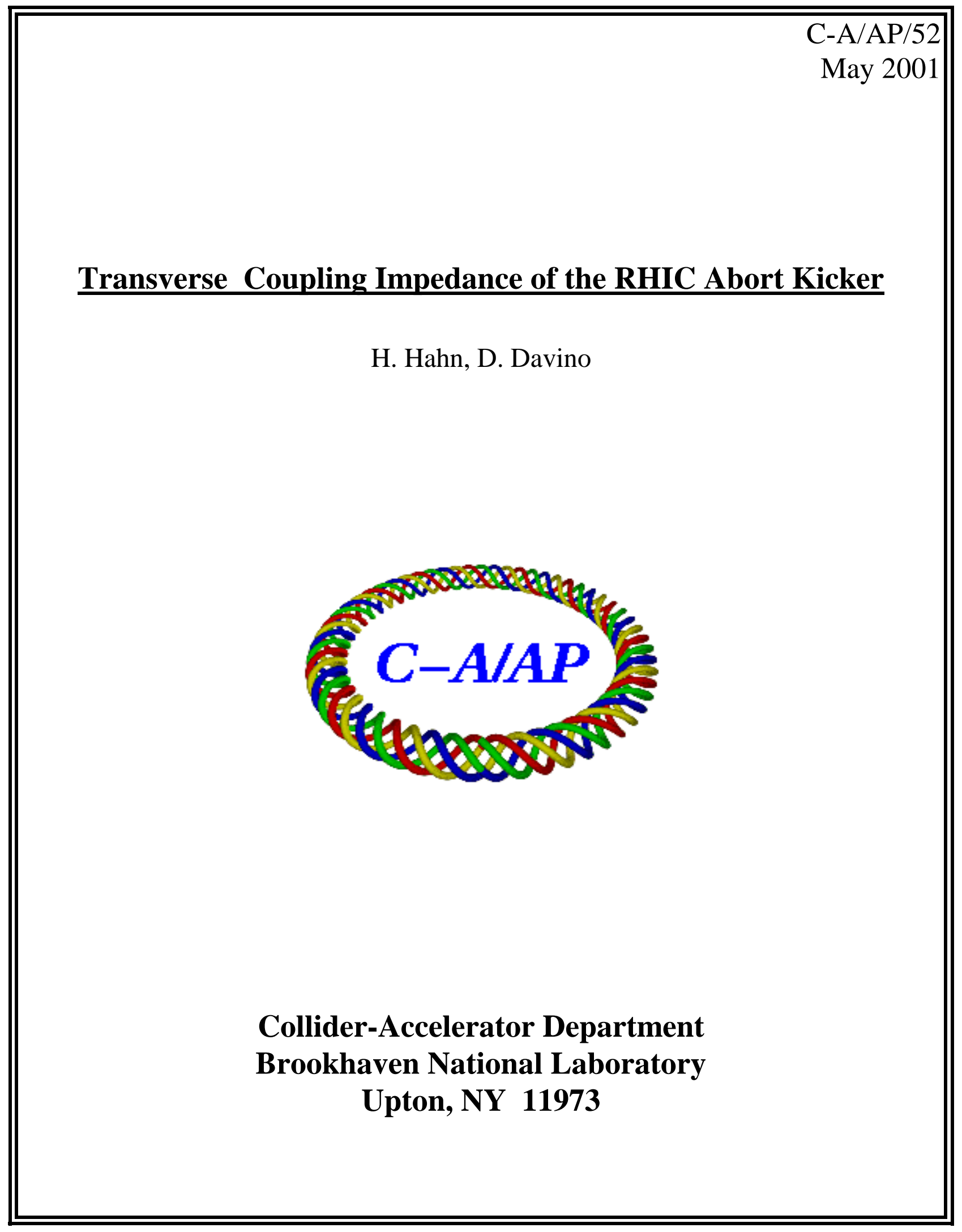




\title{
Transverse Coupling Impedance of the RHIC Abort Kicker
}

\section{H. Hahn and D. Davino}

\begin{abstract}
The results of transverse coupling impedance measurements of the RHIC abort kicker are presented. The impedance measurements were made using the standard two-wire method to obtain the forward transmission coefficient $S_{21}$ of the "Device Under Test" with respect to a reference tube of equal length, and were interpreted via the log-formula. The measurements were made in a test stand, reflecting fully the operational conditions. The transverse impedance of the lumped ferrite kicker is largest below $10 \mathrm{MHz}$ and reaches for a single unit in the horizontal, i.e. the kick direction $260+\mathrm{j} 380 \mathrm{k} \Omega / \mathrm{m}$ and in vertical direction $20+\mathrm{j} 80 \mathrm{k} \Omega / \mathrm{m}$. The contribution to the total impedance budget must reflect five kicker units per ring. At frequencies in the $\mathrm{MHz}$ range, the transverse coupling impedance in the RHIC rings is dominated by the kickers.
\end{abstract}

\section{Introduction}

The transverse coupling impedance of the RHIC rings is believed to be dominated by the resistive wall impedance and the injection and abort kickers. The resistive wall impedance has been estimated in the "two-pipe model" to be ${ }^{1}$

$$
Z_{\perp} \approx \frac{5.88}{\sqrt{n}} \mathrm{M} \Omega / \mathrm{m}
$$

with the mode number $n=\omega R / c$ and $n=1$ corresponding to the revolution frequency, $f \sim 78 \mathrm{kHz}$.

In a previous report, the results from transverse impedance measurements on the RHIC injection kicker were given. ${ }^{2}$ In this report, the results from transverse impedance measurements on a spare abort kicker unit are presented. This unit is connected to its PFN-pulser and results represent the conditions as seen by the beam. There are 5 units in each ring and the numbers per unit quoted here have to be scaled correspondingly to arrive at their total contribution to the impedance budget. In order to gain a better understanding of the impedance properties, for some measurements, the kicker was disconnected from the PFN and studied as free-standing unit.

The abort kicker is constructed as a window-frame magnet, deflecting the beam horizontally. The kicker design is given in the RHIC Design Manual, ${ }^{3}$ from where its cross section in Fig. 1 is taken. The magnet proper is assembled with CMD5005 ferrite pieces, each $20.32 \mathrm{~cm}$ long, for a total length of $122 \mathrm{~cm}$. The kicker magnet is located in a tubular vacuum vessel as shown in Fig.2. The electrical connection with the PFN pulser is through a coaxial feedthru at the middle of the magnet. 


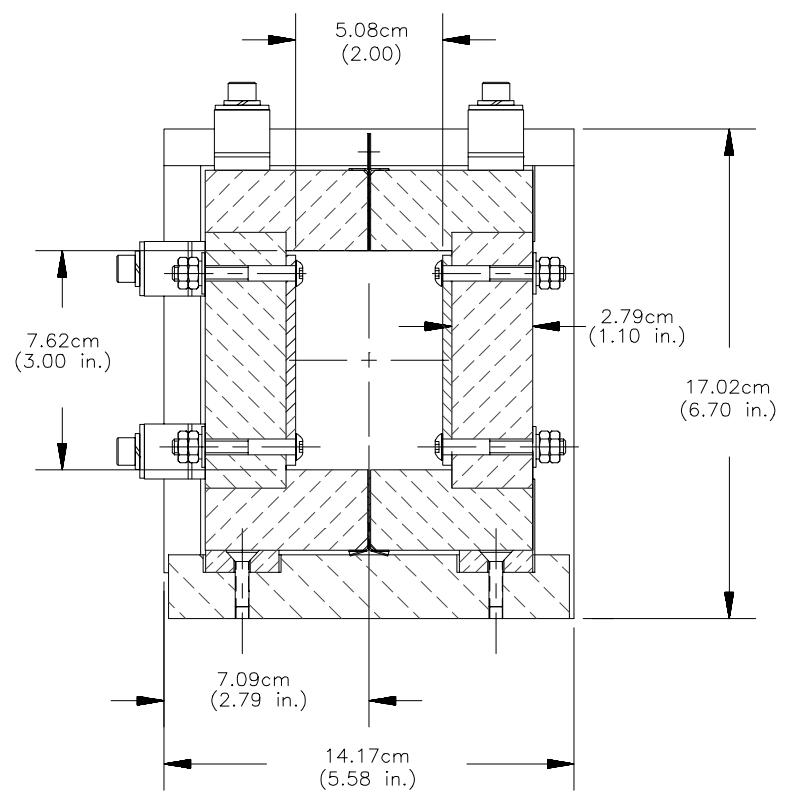

Fig.1. Abort Kicker cross section.

\section{Transverse Impedance Measurements}

Transverse impedance measurements on the RHIC injection kicker were made using the standard method, ${ }^{4}$ in which a double-wire "Lecher" line is inserted into the kicker and the forward transmission coefficients $S_{21}$ of the "Device Under Test" and of a reference line of equal length is interpreted according to

$$
Z_{D U T} \approx-2 Z_{L} \ln \left(S_{21 D U T} / S_{21 R E F}\right)
$$

from which one obtains the transverse impedance as ${ }^{5}$

$$
Z_{\perp}=\frac{c Z_{D U T}}{\omega \Delta^{2}}=-2 \frac{c Z_{L}}{\omega \Delta^{2}} \ln \left(S_{21 D U T} / S_{21 R E F}\right)
$$

with $\Delta$ being the spacing of the two wires and $Z_{L}$ the characteristic impedance of the line. In order to obtain sufficiently strong signals, measurements were made with a commercial $450 \Omega$ "Ladder" cable CQ551, which has a spacing of $\Delta=13 / 16$ in. $=2.064$ $\mathrm{cm}$. Matching of the $450 \Omega$ line to the $50 \Omega$ of the network analyzer, Agilent $8753 \mathrm{ES}$, is achieved by means of a transformer with a center-tapped secondary winding which serves as $180^{\circ}$ hybrid. The transformer at the input has a 50/450 ratio whereas the output transformer has a ratio of $300 / 50$ and resistive matching by two $75 \Omega$ resistors was applied thereby covering frequencies up to $\sim 100 \mathrm{MHz}$. Common mode suppression was achieved by measuring $Z_{D U T}$ twice, the second time with the line rotated by $180^{\circ}$, and all results quoted represent the average of these two measurements. All measurements were made with the network analyzer set for a logarithmic frequency scale from $30 \mathrm{kHz}$ to 100 $\mathrm{MHz}, 1601$ points, a $100 \mathrm{~Hz}$ bandwidth, and averaged over 3 sweeps. The transformers 
are rated only for frequencies above $100 \mathrm{kHz}$, and measurement results at lower frequencies become suspect and must be used with care. In fact, the results presented here are given only for frequencies above $500 \mathrm{kHz}$.

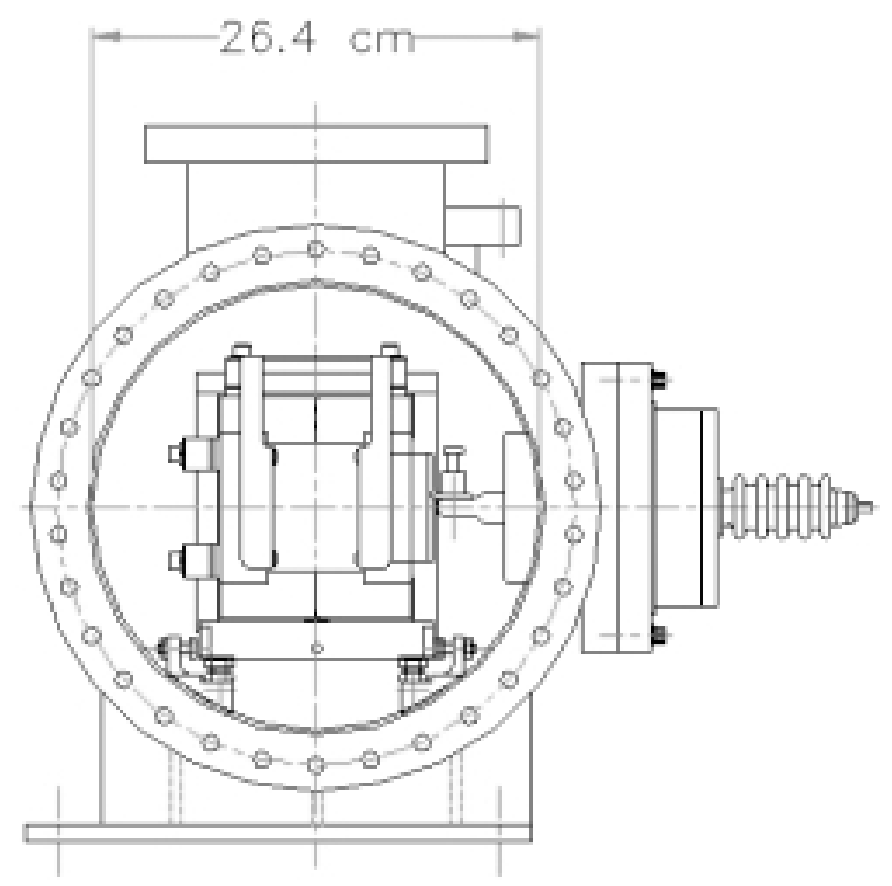

Fig. 2. Side view of Abort Kicker with vacuum vessel and feed thru.

\section{Experimental Results}

The measurements were performed on a abort kicker unit in a test stand fully reflecting the operational conditions, i.e. with the PFN pulser connected. The results for the vertical transverse impedance are shown in Fig.3.

The results for the horizontal (in kick direction) transverse coupling impedance are shown in Figs. 4 and 5 with and without the PFN pulser. The results with PFN can be summarized by

$$
\begin{aligned}
& Z_{\perp V} \leq(20+j 80) \\
& Z_{\perp H} \leq(260+j 380)
\end{aligned} \mathrm{k} \Omega / \mathrm{m} ; f>5 \mathrm{MHz} .
$$

It follows that at frequencies in the $\mathrm{MHz}$ range the transverse impedance is dominated by the kickers. It is worth pointing out that the PFN, although only capacitively coupled via the non-conducting thyratron, is clearly visible in the impedance and that the PFN terminating resistor of $\sim 2 \Omega$, in general, lowers the coupling impedance. The effect of an external load, represented by a $25 \Omega$ load or a short, is visualized in Figs. 6 and 7 . It is to be noted that the measurements cannot be reconciled with the simple Nassibian-Sacherer model for a window frame magnet. ${ }^{5}$ At frequencies below $\sim 5 \mathrm{MHz}$, the real part of the 
impedance behaves contrary to theoretical predictions and could be a result of the small signal to noise ratio, but further studies are indicated.
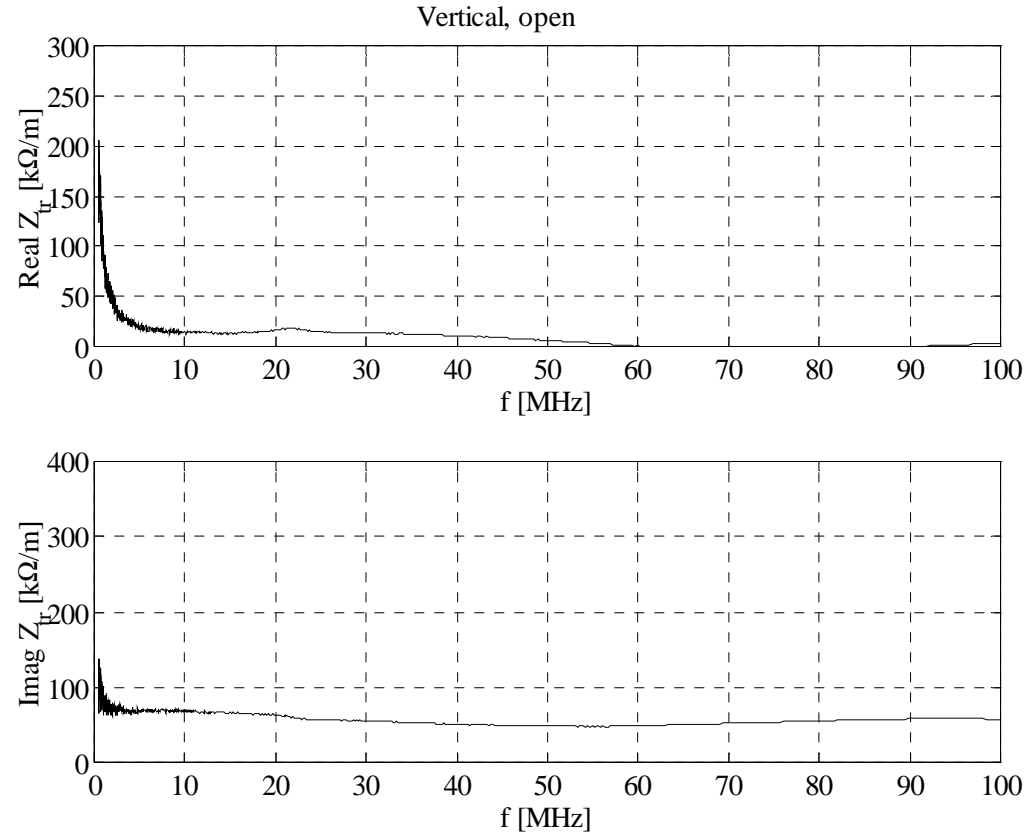

Fig.3. Vertical transverse coupling impedance of RHIC abort kicker.
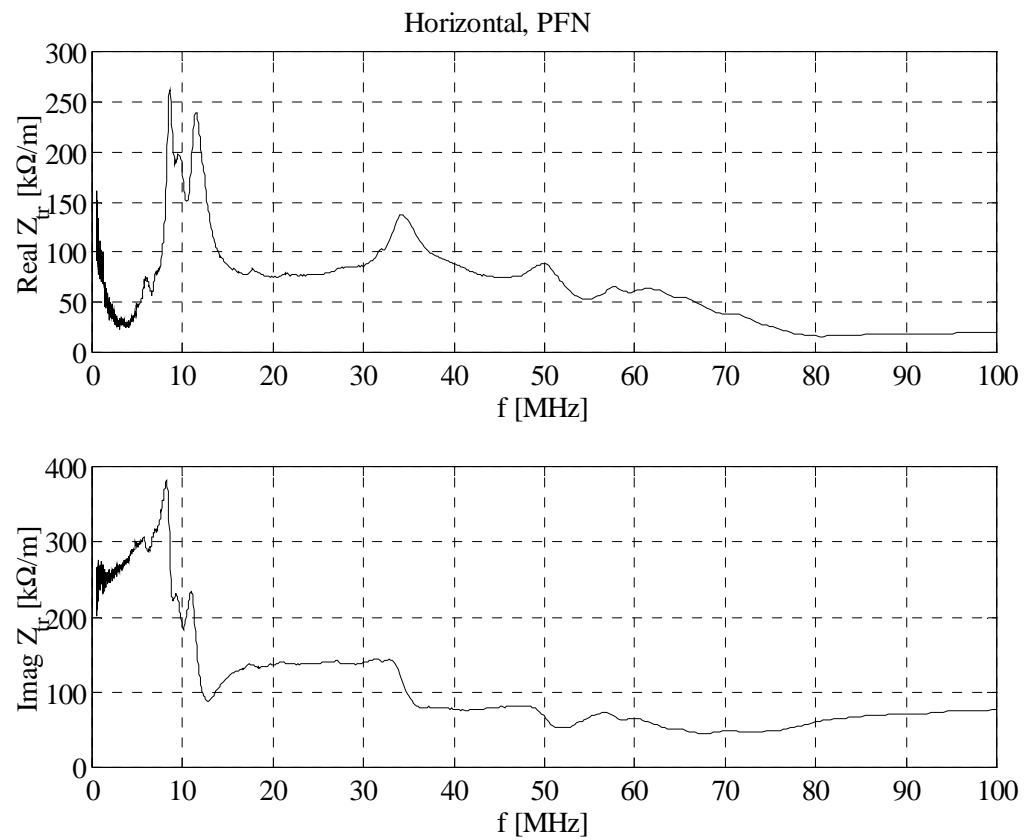

Fig. 4. Horizontal transverse coupling impedance of RHIC Abort Kicker (with PFN). 

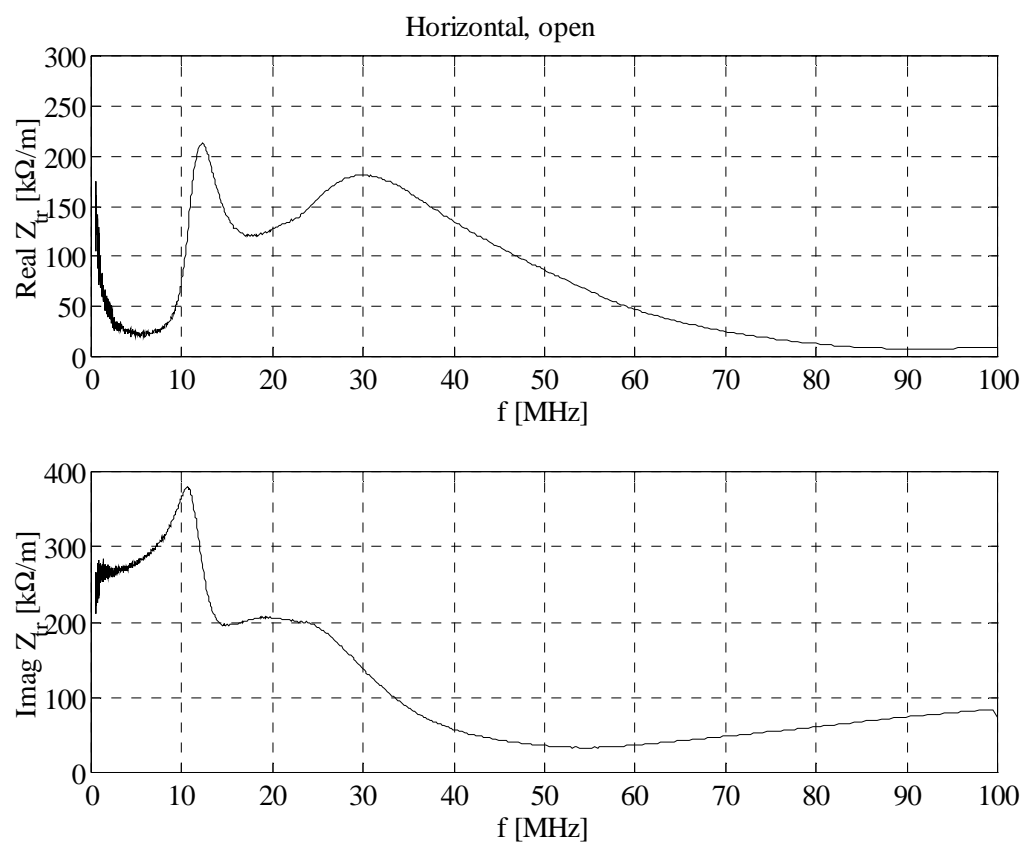

Fig.5. Horizontal transverse coupling impedance of RHIC Abort kicker (w.o. PFN).
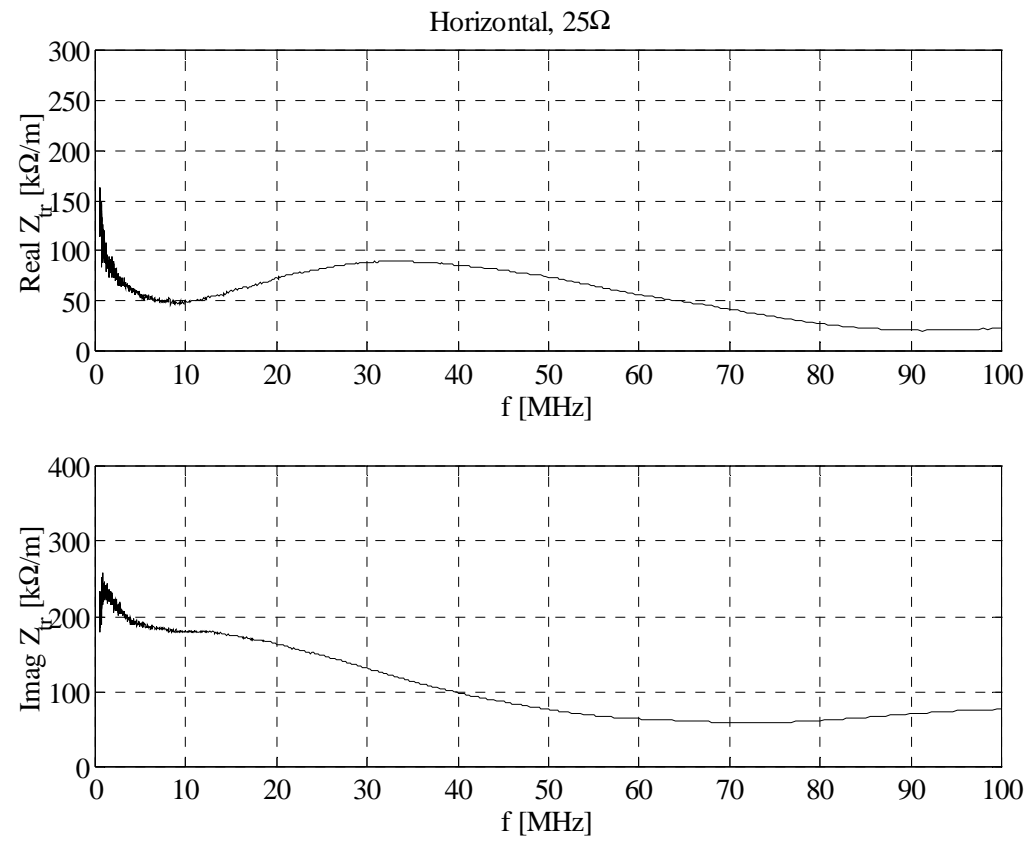

Fig. 6. Horizontal coupling impedance with $25 \Omega$ termination. 

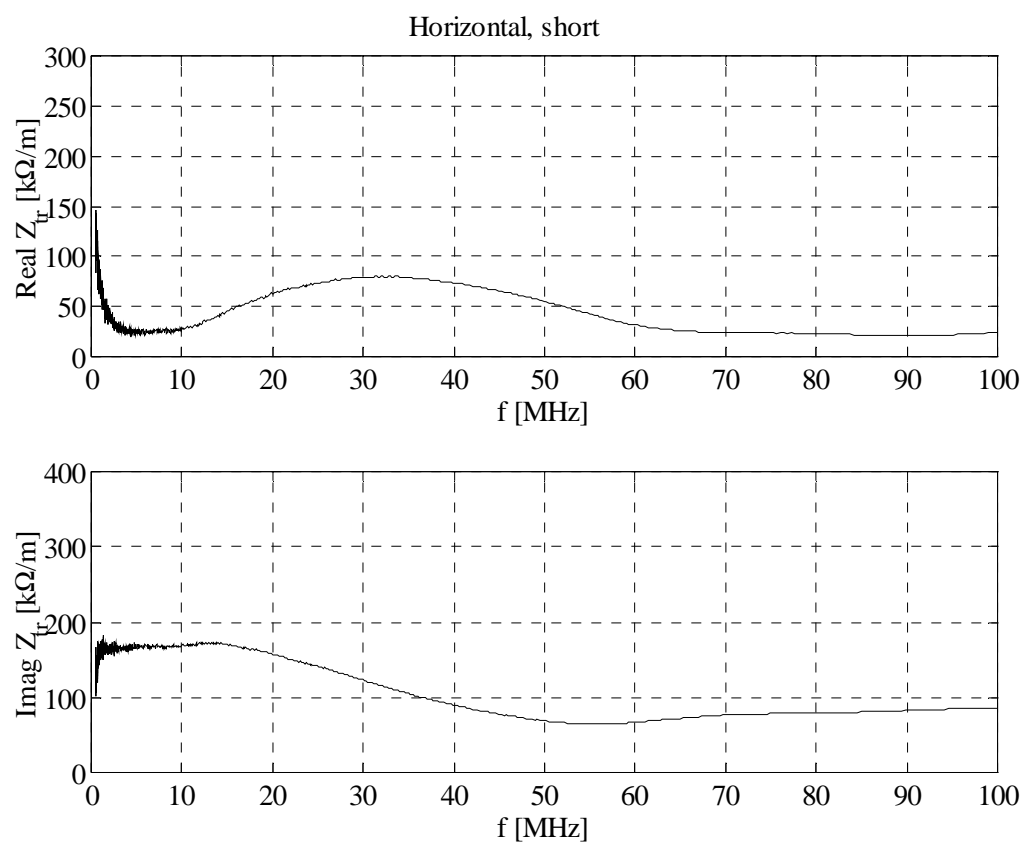

Fig.7. Horizontal coupling impedance with shorted termination.

\section{Electromagnetic Field Analysis}

Difficulties encountered in the measurement of the transverse coupling impedance at low frequencies pointed to the need for analytic calculations. In view of the fact that the impedance perpendicular to the kick direction is essentially independent of the load, its field analysis can be based on the simplified kicker model shown in Fig.8. (Note that the directions in the figure are rotated with respect to the abort kicker). The analysis of the impedance in kick direction will be presented in a separate report

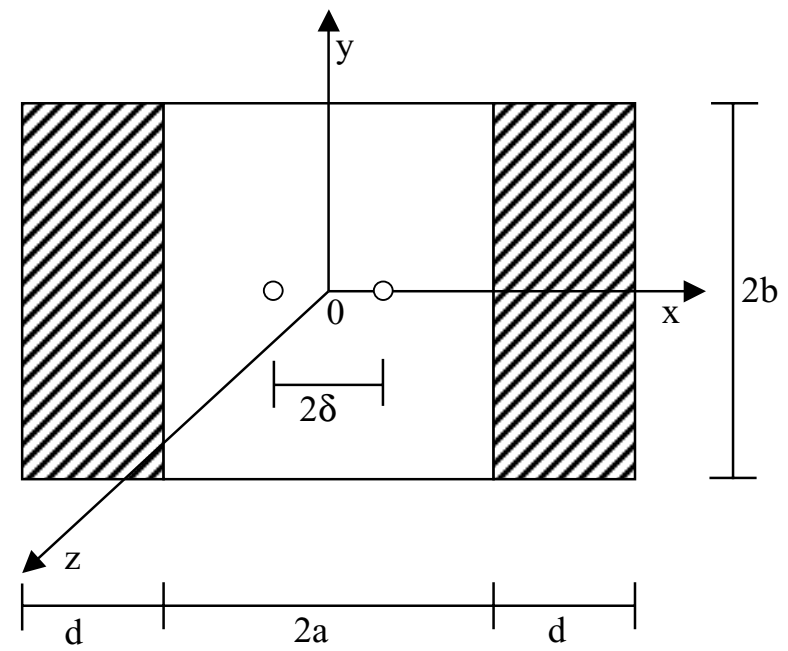

Fig. 8. Simplified Kicker Model used for the analytical calculations. 
The field analysis is here performed via the standard field matching procedure involving the transverse fields at the vacuum-ferrite interface. In the limit of ultrarelativistic beams the dipole current generates pure transverse fields which can be represented by the twin-conductor cable. Assuming unit-strength current, a wire spacing of $\Delta=2 \delta$, and by omitting the common factor $\left\{e^{-j k z+j \omega t}\right\}$ one obtains the source fields ${ }^{6}$ (with natural units, $c=1, \mu_{0}=1$, whence $\omega=k$ )

$$
\begin{aligned}
& E_{y}^{S}=\frac{1}{2 b}\left[\frac{\sin \eta y \cosh \eta(x-\delta)}{\cosh 2 \eta(x-\delta)-\cos 2 \eta y}-\frac{\sin \eta y \cosh \eta(x+\delta)}{\cosh 2 \eta(x+\delta)-\cos 2 \eta y}\right]\{\} \\
& H_{y}^{S}=\frac{1}{2 b}\left[\frac{\cos \eta y \sinh \eta(x-\delta)}{\cosh 2 \eta(x-\delta)-\cos 2 \eta y}-\frac{\cos \eta y \sinh \eta(x+\delta)}{\cosh 2 \eta(x+\delta)-\cos 2 \eta y}\right]\{\}
\end{aligned}
$$

with $\eta=\pi /(2 b)$ and corresponding expressions for $E_{x}$ and $H_{x}$. Field matching at the interface requires the additional fields in the beam region,

$$
\begin{aligned}
& E_{x}=\sum_{n} c_{e n}\left(\eta_{n}^{2}+k^{2}\right) \cosh \eta_{n} x \cos \eta_{n} y \\
& E_{y}=-\sum_{n}\left(c_{e n} \eta_{n}^{2}+c_{h n} k^{2}\right) \sinh \eta_{n} x \sin \eta_{n} y \\
& E_{z}=j k \sum_{n}\left(c_{h n}-c_{e n}\right) \eta_{n} \sinh \eta_{n} x \cos \eta_{n} y \\
& H_{y}=\sum_{n}\left(c_{h n} \eta_{n}^{2}+c_{e n} k^{2}\right) \cosh \eta_{n} x \cos \eta_{n} y \\
& H_{z}=j k \sum_{n}\left(c_{e n}+c_{h n}\right) \eta_{n} \cosh \eta_{n} x \sin \eta_{n} y
\end{aligned}
$$

and in the ferrite region,

$$
\begin{aligned}
& E_{y}^{F}=-\sum_{n}\left(\mu c_{h n}^{F} k^{2}+c_{e n}^{F} \kappa_{n} \eta_{n}\right) \sinh \kappa_{n}(x-a-d) \sin \eta_{n} y \\
& E_{z}^{F}=j k \sum_{n}\left(\mu c_{h n}^{F} \eta_{n}-c_{e n}^{F} \kappa_{n}\right) \sinh \kappa_{n}(x-a-d) \cos \eta_{n} y \\
& H_{y}^{F}=\sum_{n}\left(c_{h n}^{F} \kappa_{n} \eta_{n}-\varepsilon c_{e n}^{F} k^{2}\right) \cosh \kappa_{n}(x-a-d) \cos \eta_{n} y \\
& H_{z}^{F}=j k \sum_{n}\left(\varepsilon c_{e n}^{F} \eta_{n}-c_{h n}^{F} \kappa_{n}\right) \cosh \kappa_{n}(x-a-d) \sin \eta_{n} y
\end{aligned}
$$

with the wave numbers related by

$$
\kappa_{n}^{2}=\eta_{n}^{2}-(\varepsilon \mu-1) k^{2} \text { and } \eta_{n}=(2 n-1) \eta ; n=1,2,3, \ldots
$$

The formal expression for the transverse coupling impedance is defined as

$$
Z_{\perp}=\frac{j}{2 \delta} \sum_{n} \eta_{n}^{2}\left(c_{e n}-c_{h n}\right)
$$


with the expansion coefficients determined via the macsyma program

$$
\begin{aligned}
c_{e n} & =\frac{\varepsilon P_{e n} \eta_{n} \cosh \kappa_{n} d-P_{h n} \kappa_{n} \sinh \kappa_{n} d}{\left(\eta_{n}^{2}+k^{2}\right)\left(\varepsilon \eta_{n} \cosh \kappa_{n} d \sinh \eta_{n} a+\kappa_{n} \sinh \kappa_{n} d \cosh \eta_{n} a\right)} \\
c_{h n}= & \frac{P_{e n} \kappa_{n} \cosh \kappa_{n} d-\mu P_{h n} \eta_{n} \sinh \kappa_{n} d}{\left(\eta_{n}^{2}+k^{2}\right)\left(\kappa_{n} \cosh \kappa_{n} d \sinh \eta_{n} a+\mu \eta_{n} \sinh \kappa_{n} d \cosh \eta_{n} a\right)}
\end{aligned}
$$

The $P_{e}$ and $P_{h}$ terms appearing above represent the shorthand symbols

$$
\begin{aligned}
& P_{e n}=\frac{1}{b} \int_{-b}^{b} d y E_{y}^{S}(x=a) \sin \eta_{n} y \\
& P_{h n}=\frac{1}{b} \int_{-b}^{b} d y H_{y}^{S}(x=a) \cos \eta_{n} y
\end{aligned}
$$

The dipole source field at the interface, $x=a$, follows by Taylor expansion as

$$
\begin{aligned}
& E_{y}^{s}=\frac{\eta \delta}{2 b}\left[\frac{(\sinh 3 \eta a+2 \sinh \eta a) \sin \eta y+\sinh \eta a \sin 3 \eta y}{(\cosh 2 \eta a-\cos 2 \eta y)^{2}}\right] \\
& H_{y}^{s}=\frac{\eta \delta}{2 b}\left[\frac{(\cosh 3 \eta a-2 \cosh \eta a) \cos \eta y+\cosh \eta a \cos 3 \eta y}{(\cosh 2 \eta a-\cos 2 \eta y)^{2}}\right] .
\end{aligned}
$$

The shorthand $P$ symbols can now be written as

$$
\begin{aligned}
& P_{e n}=F_{1 e} S_{1 e n}+F_{3 e} S_{3 e n} \\
& P_{h n}=F_{1 h} S_{1 h n}+F_{3 h} S_{3 h n}
\end{aligned}
$$

with the factors (note that $\eta=\eta_{1}$ )

$$
\begin{aligned}
& F_{1 e}=\frac{\eta}{2 b} \frac{\sinh 3 \eta a+2 \sinh \eta a}{\cosh ^{2} 2 \eta a} \delta \\
& F_{3 e}=\frac{\eta}{2 b} \frac{\sinh \eta a}{\cosh ^{2} \eta a} \delta \\
& F_{1 h}=\frac{\eta}{2 b} \frac{\cosh 3 \eta a-2 \cosh \eta a}{\cosh ^{2} 2 \eta a} \delta \\
& F_{3 h}=\frac{\eta}{2 b} \frac{\cosh \eta a}{\cosh ^{2} 2 \eta a} \delta
\end{aligned}
$$

and the integrals 


$$
\begin{aligned}
& S_{m e n}=\frac{1}{b} \int_{-b}^{b} d y \frac{\cosh ^{2} 2 \eta a \sin m \eta y \sin \eta_{n} y}{(\cosh 2 \eta a-\cos 2 \eta y)^{2}} \\
& S_{m h n}=\frac{1}{b} \int_{-b}^{b} d y \frac{\cosh ^{2} 2 \eta a \cos m \eta y \cos \eta_{n} y}{(\cosh 2 \eta a-\cos 2 \eta y)^{2}}
\end{aligned}
$$

The ferrite properties, $\varepsilon$ and $\mu$, enter the expressions only via the $\kappa$ wave number in $\mathrm{x}$ direction but not the $P$ - integrals. The ferrite material CMD5005 has a dielectric constant $\varepsilon=12$. Measured values for the permeability were made available by the vendor. ${ }^{7}$ In Fig 7 measured values and values computed from a frequently used expression ${ }^{8}$

$$
\mu=1+\frac{\mu_{i}-1}{1+j \omega \tau}
$$

with $\mu_{\mathrm{I}}=1830$ and $\tau=1 /(2 \pi 2.23 \mathrm{MHz})$ are compared.
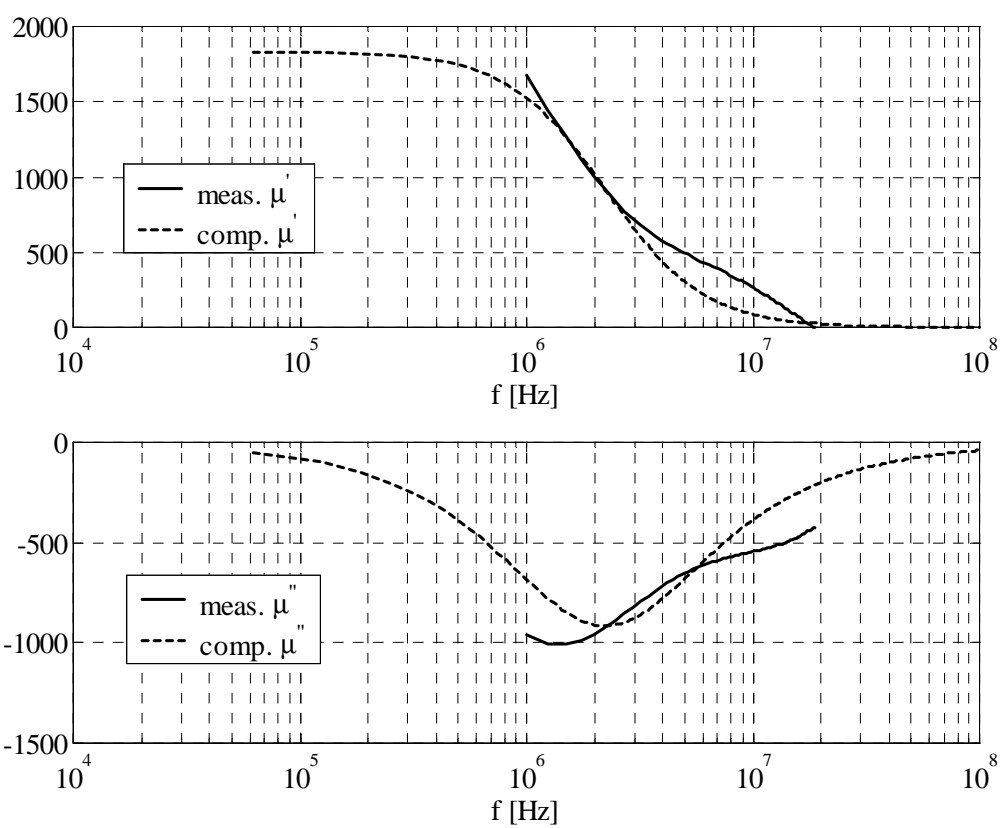

Fig.9. Measured and computed permeability of CMD5005.

Values for the $P$-terms have been found by numerical integration taking the actual spacing, $\Delta$, of the wires and were used in the computation of the coupling impedance shown in Fig.10. It is to be noted that the results are essentially equal for the measured and computed permeabilities. General agreement of the computed results in Fig. 10 with the measurements in Fig.3 is found, although the real part is smaller and vanishes in the limit of low frequencies.

In Fig.10 the results from numerical integration are also compared to those obtained by Taylor expansion and $\delta \rightarrow 0$. In this limit, inspection of the integrals suggests that for the case with $a \geq b$, a first order approximation for the transverse 
coupling impedance is obtained by retaining only $\mathrm{n}=1$ with $S_{3 e 1}=S_{3 h 1} \approx 0$ and $S_{1 e 1}=S_{1 h 1} \approx 1$. Since the beam oscillations are assumed to be small, the $\delta \rightarrow 0$ case is applicable, and one can draw the conclusion that the measurements with a cable size filling half the aperture yield coupling impedance values too high by $\sim 10 \%$.
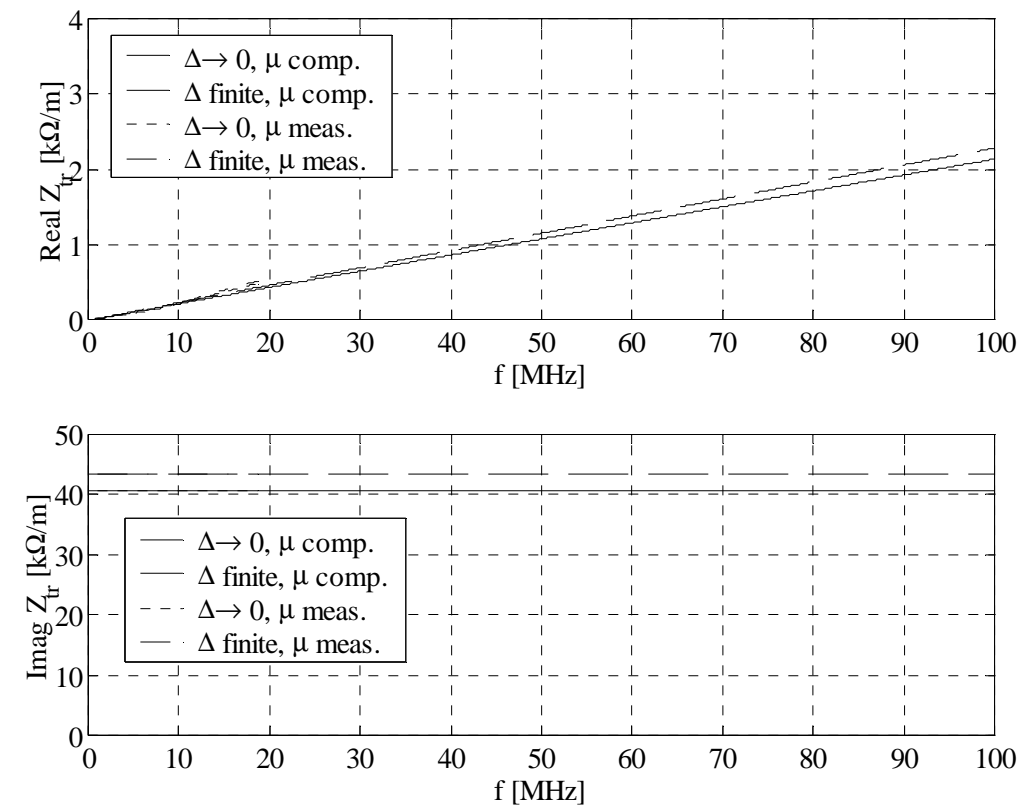

Fig.10 Computed vertical abort kicker coupling impedance.

\section{Acknowledgements}

The authors would like to acknowledge discussions with. M.M. Blaskiewicz and the engineering help from C. Pai and the technical support from K. Hartmann and J. Cupolo.

\section{References}

${ }^{1}$ S. Peggs and W.W. MacKay, eds. Report RHIC/AP/36, (BNL, September 1994).

${ }^{2}$ H. Hahn and D. Davino, Technical Report C-A/AP/45, (BNL, March 2001).

${ }^{3}$ RHIC Design Manual, http://www.rhichome.bnl.gov/NT-share/rhicdm/6_bd2a.pdf.

${ }^{4}$ F. Caspers, in Handbook of Accelerator Physics and Engineering, ed. A.W. Chao and M. Tigner, p.571.

${ }^{5}$ Nassibian and Sacherer, Nucl. Instr. \& Methods, 159, 21 (1979).

${ }^{6}$ H. Tsutsui, Report CERN-SL-2000 AP (CERN, January 2000).

${ }^{7}$ Courtesy of G.E. Schaller at Ceramic Magnetics, Inc..

${ }^{8}$ L. Vos, Report CERN-SL-2000-010 AP (March 2000). 